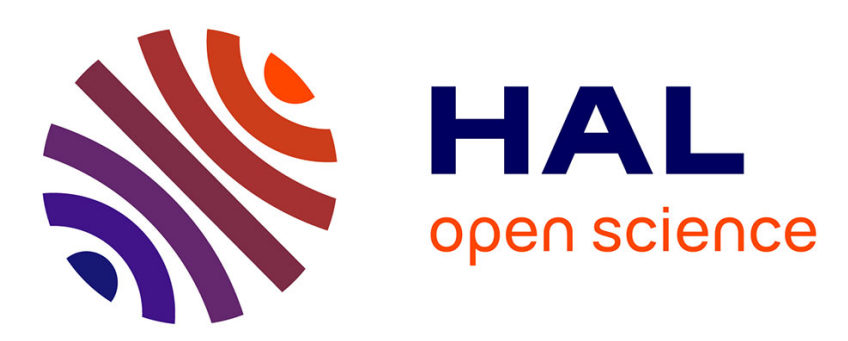

\title{
An experimental and analytical study of the elasticity of model polyurethane networks crosslinked by tri- and quadriisocyanate
}

\author{
Bruno Fayolle, Pierre Gilormini, Julie Diani
}

\section{- To cite this version:}

Bruno Fayolle, Pierre Gilormini, Julie Diani. An experimental and analytical study of the elasticity of model polyurethane networks crosslinked by tri- and quadriisocyanate. Colloid and Polymer Science, 2010, 288, pp.97-103. 10.1007/s00396-009-2145-6 . hal-00794193

\section{HAL Id: hal-00794193 \\ https://hal.science/hal-00794193}

Submitted on 25 Feb 2013

HAL is a multi-disciplinary open access archive for the deposit and dissemination of scientific research documents, whether they are published or not. The documents may come from teaching and research institutions in France or abroad, or from public or private research centers.
L'archive ouverte pluridisciplinaire $\mathbf{H A L}$, est destinée au dépôt et à la diffusion de documents scientifiques de niveau recherche, publiés ou non, émanant des établissements d'enseignement et de recherche français ou étrangers, des laboratoires publics ou privés. 


\title{
An experimental and analytical study of the elasticity of model polyurethane networks crosslinked by tri- and quadriisocyanate
}

\author{
Bruno Fayolle · Pierre Gilormini · Julie Diani
}

\begin{abstract}
Polyurethane networks have been prepared from a mix of tri- and quadriisocyanate and from two types of diols, polyether-based (with molar masses of $1,000,2,000$, and 4,000 g/mol) and polyester-based $(1,035 \mathrm{~g} / \mathrm{mol})$. The weight fraction of sol has been measured, as well as the elastic shear modulus of the gels. It has been found that the statistical theory of network formation predicts a weight fraction of sol in agreement with the experimental results, but its standard combination with the theory of rubber elasticity disagrees significantly with the elastic modulus measured. This suggests a discrepancy between theory and experiment in terms of elastically active chains. In contrast, the assumption that all nodes in the gel, or even in the system, are elastically active gives much better predictions for the system considered.
\end{abstract}

Keywords Polyurethane - Model networks • Rubber elasticity

\section{Introduction}

The relation between the structure of elastomers and their elastic properties has been the subject of numerous investigations, and an appealing way to test the various theories is the use of model networks. Polyurethane elastomers are good candidates for such studies, they offer a variety of possible systems and have been widely used, as mentioned in [1], for instance. To cite a few among many examples, triols and

B. Fayolle $(\varangle) \cdot$ P. Gilormini · J. Diani

Laboratoire d'Ingénierie des Matériaux, CNRS,

Arts et Metiers ParisTech, 151 Blvd de l'Hôpital, 75013 Paris, France

e-mail: bruno.fayolle@paris.ensam.fr diisocyanate were used in [2], mono-, bi-, and trifunctional polyols were reacted with diisocyanate in [3], whereas diols and triisocyanate were considered in [4]. In this paper, networks were prepared from two types of diols reacted with a mixture of triisocyanate and quadriisocyanate, for various molar ratios of $\mathrm{NCO}$ and $\mathrm{OH}$ groups, with an excess of the latter. This produced non-ideal networks, the elastic properties of which were measured and compared to the predictions of standard models.

Two models must be combined to relate the chemical composition of a network to its elastic properties, actually. A first model is required to deduce such features of the network as the crosslink density, for instance, which vary with the functionality of the components, with stoichiometry, and with conversion ratio, among other factors. In this study, the results given by the statistical network formation. Theory were obtained by using probability-generating functions [5]. A second model is necessary to deduce the mechanical behavior from the microstructure, such as the elementary theory of rubber elasticity [6], or the more elaborate phantom network theory [7], with possible account for inter-chain entanglements [8-11]. All these models fail to predict the experimental results obtained with our systems, and variations are proposed eventually to get better agreement.

\section{Experimental}

Materials

Two types of polyurethane networks were prepared in solution, following a procedure proposed by 
Pegoraro et al. [12]. The first type of network used poly(propylether), with three different molar masses considered $(1,000,2,000$, and 4,000 $\mathrm{g} / \mathrm{mol})$, while the second, polyester based, used poly(tetramethylene adipate) with a molar mass of $1,035 \mathrm{~g} / \mathrm{mol}$. The poly(propylether) (PE) and poly(tetramethylene adipate) (PTMA) were kindly supplied by Bayer (Desmophen 1110, 2062, and 4027) and by COIM (Diexter 215), respectively.

An isocyanate supplied by Rhodia (Tolonate HDT) was used for crosslinking. In order to avoid side reactions with water molecules, it was dried under vacuum, then dissolved in twice its volume of ethyl acetate, and the mixture was kept under nitrogen. The isocyanate appeared to be a mix of tri- and quadriisocyanate, as could be deduced from gel permeation chromatography that inferred a weight average functionality of $\bar{f}_{w}=3.4$. In order to determine the molar fractions $\varphi$ and $1-\varphi$ of triisocyanate and quadriisocyanate in the isocyanate mix, one can start from the definition

$\bar{f}_{w}=3 m_{I 3}+4 m_{I 4}$,

where $m_{I 3}$ and $m_{I 4}$ denote the weight fractions of triisocyanate and quadriisocyanate, respectively, with $m_{I 3}+$ $m_{I 4}=1$. For triisocyanate, we have $m_{I 3}=\varphi M_{I 3} / \bar{M}_{I}$, where $\bar{M}_{I}=\varphi M_{I 3}+(1-\varphi) M_{I 4}$ is the average number molar mass of the isocyanate mix, and for quadriisocyanate, we have $m_{I 4}=(1-\varphi) M_{I 4} / \bar{M}_{I}$. Therefore, the weight average functionality writes

$\bar{f}_{w}=\frac{3 \varphi M_{I 3}+4(1-\varphi) M_{I 4}}{\varphi M_{I 3}+(1-\varphi) M_{I 4}}$,

which leads to the molar fraction of triisocyanate:

$\varphi=\left(1+\frac{M_{I 3}}{M_{I 4}} \frac{\bar{f}_{w}-3}{4-\bar{f}_{w}}\right)^{-1}$.

Using $\bar{f}_{w}=3.4$ and assuming that a molecule of quadriisocyanate is obtained by reaction between two molecules of triisocyanate, with one molecule of carbon dioxide produced, i.e., $M_{I 3}=504.6 \mathrm{~g} / \mathrm{mol}$ (as given by the supplier) and $M_{I 4}=965.2 \mathrm{~g} / \mathrm{mol}$; this equation gives $\varphi=0.74$. Alternatively, starting from the weight fraction $w_{\mathrm{NCO}}$ of $\mathrm{NCO}$ (with a molar mass of $M_{\mathrm{NCO}}=$ $42 \mathrm{~g} / \mathrm{mol}$ ) in the mixture, one can write

$w_{\mathrm{NCO}}=\frac{(4-\varphi) M_{\mathrm{NCO}}}{\varphi M_{I 3}+(1-\varphi) M_{I 4}}$, which leads to the following expression for the fraction of triisocyanate:

$\varphi=\frac{M_{I 4} w_{\mathrm{NCO}}-4 M_{\mathrm{NCO}}}{\left(M_{I 4}-M_{I 3}\right) w_{\mathrm{NCO}}-M_{\mathrm{NCO}}}$,

which gives $\varphi$ between 0.69 and 0.80 for the weight fraction of NCO, $22 \pm 0.5 \%$, provided by the supplier. This range is consistent with the $\varphi=0.74$ value obtained above, and that will be used in the sequel.

By varying the masses of diol and isocyanate mix used for crosslinking reaction, a set of ratios between the number of moles of the two functional groups $r=[\mathrm{NCO}] /[\mathrm{OH}]$ could be obtained. This ratio is easily related to the molar fractions $n_{D}$ and $n_{I}$ of diol and isocyanate, respectively:

$r=\frac{4-\varphi}{2} \frac{n_{I}}{n_{D}}$,

where coefficient 2 in the denominator renders the bifunctionality of the diols. In this study, only $r \leq 1$ values are considered, i.e., there is an excess of $\mathrm{OH}$ groups and the reaction is completed when all $\mathrm{NCO}$ groups have reacted. Thus, denoting by $x$ the fraction of reacted NCO groups, the reaction proceeds from $x=0$ to $x=1$, with a fraction $r x$ of $\mathrm{OH}$ groups being reacted, and with a fraction $1-r$ of $\mathrm{OH}$ groups that remain unreacted eventually. Of course, this residual fraction is zero when $r=1$, i.e., for stoichiometric conditions. The following relations, which result from Eq. 6 and from $n_{D}+n_{I}=1$, will be useful when models are applied:

$n_{D}=\frac{4-\varphi}{2 r+4-\varphi} \quad$ and $\quad n_{I}=\frac{2 r}{2 r+4-\varphi}$.

Finally, it will also be convenient to define the weight fraction of diols

$m_{D}=\frac{n_{D} M_{D}}{\bar{M}}=\left(1+\frac{2 r}{4-\varphi} \frac{\bar{M}_{I}}{M_{D}}\right)^{-1}$,

where the rightmost expression is obtained from Eq. 6, with

$$
\begin{aligned}
\bar{M} & =n_{D} M_{D}+n_{I} \bar{M}_{I} \\
& =n_{D} M_{D}+n_{I} \varphi M_{I 3}+n_{I}(1-\varphi) M_{I 4}
\end{aligned}
$$

denoting the average molar mass of the mixture.

Sample preparation and mechanical behavior

For the PE diol, the following procedure was used. First, the diol was mixed for 3 min under vacuum at 
$30^{\circ} \mathrm{C}$ with $1 \%$ by weight of dibutyletin dilaurate used as a catalyst. Then, isocyanate was added and the mixture was poured into a Petri dish and kept at $60^{\circ} \mathrm{C}$ for $24 \mathrm{~h}$. For the PTMA diol, the procedure was different because of its trend to crystallize: the diol was dissolved in an equal weight of ethyl acetate in a flat-bottomed flask with a mechanical stirrer, and the mixture was poured into a Petri dish and kept at $60^{\circ} \mathrm{C}$ for $24 \mathrm{~h}$. Complete evaporation of the solvent was obtained after this period. In both cases, polyurethane films of less than 1-mm thickness were obtained and the presence of unreacted $\mathrm{NCO}$ (at 2,256 $\mathrm{cm}^{-1}$ ) groups was analyzed by Fourier transform infrared in attenuated total reflectance mode with a resolution of $4 \mathrm{~cm}^{-1}$, using a Bruker IFS 28 spectrophotometer. This confirmed that the conversion was completed, within the precision of spectrophotometry, after $24 \mathrm{~h}$. For the PTMA diol, the weight fraction of the sol was measured for various $r$ values by extracting and weighting the gel phase. These results are reported in the next section.

The viscosity of the polyether-based polyurethanes has been measured at $40^{\circ} \mathrm{C}$ with an ARES rheometer from TA Instruments, using parallel plates with a diameter of $25 \mathrm{~mm}$. A good sensitivity was obtained with a strain amplitude of 0.20 . For the 1,000 - and $2,000-\mathrm{g} / \mathrm{mol}$ diols, viscosities of 0.06 and $0.12 \mathrm{~Pa}$ s were measured, but the $4,000-\mathrm{g} / \mathrm{mol}$ diol departed from this linear law with a viscosity of $0.35 \mathrm{~Pa} \mathrm{~s}$, which suggests a molar mass between entanglements between 2,000 and $4,000 \mathrm{~g} / \mathrm{mol}$. The Young modulus $E$ of dumbbellshaped specimens ( $25 \mathrm{~mm}$ long and $4 \mathrm{~mm}$ wide) cut from the unextracted polyurethane films was measured at room temperature on a tensile testing machine Instron 4301 with a $100 \mathrm{~N}$ load cell and a constant crosshead velocity of $10 \mathrm{~mm} / \mathrm{min}$. A linear relation between the force per unit unstrained area and the parameter $\lambda-1 / \lambda^{2}$, where $\lambda$ denotes axial extension ratio, was obtained up to $\lambda$ values of about $2(100 \%$ extension). The shear modulus $G$ was obtained from $E$ by assuming incompressibility, i.e., $G=E / 3$, and is shown in Fig. 1 with standard deviation for a series of three to four specimens. As expected, it can be observed that different diols with the same molar mass lead to very similar shear moduli and that smaller shear moduli are obtained for larger molar masses. The figure suggests that gelation occurs for $r$ about 0.35 , and it was noted indeed during the experiments that mixtures prepared with $r$ lower than this value kept liquid at the end of the reaction, whatever the diol molar mass. More interestingly, a downward curvature is observed in Fig. 1, whereas an upward curvature is usually reported for polyurethanes. This is discussed in the following section.

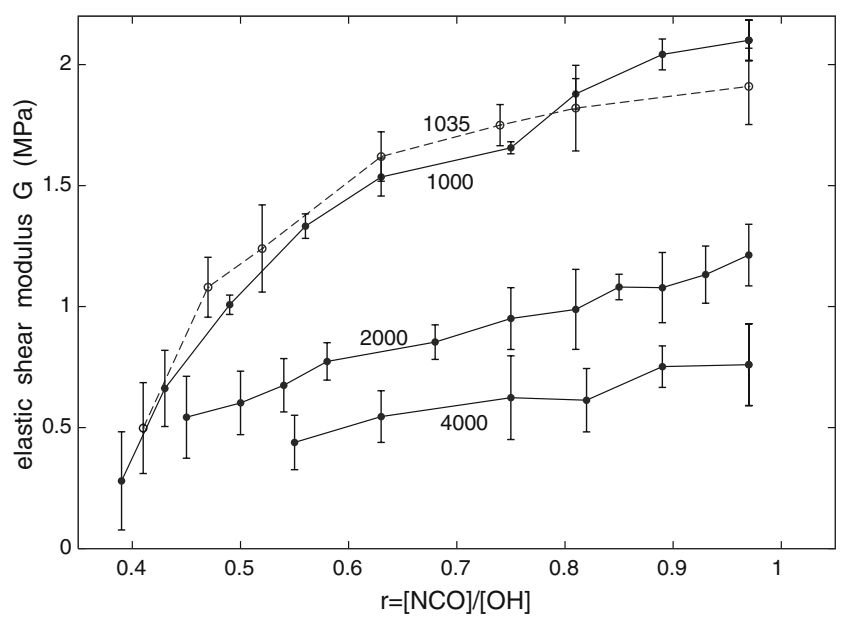

Fig. 1 Elastic shear modulus measured on the various polyurethane networks prepared from polyether diol (full symbols and unbroken lines, with molar masses as indicated, in $\mathrm{g} / \mathrm{mol}$ ), or from polyester diol (open symbols and broken line, with a molar mass of $1,035 \mathrm{~g} / \mathrm{mol}$ )

\section{Model and discussion}

\section{Network formation}

The theory of network formation has been developed long ago from the theory of stochastic processes. Following the pioneering works of Flory [13] and Stockmayer [14], its further developments used the theory of stochastic branching processes, with two main variants. The first one [5] uses probability-generating functions, whereas the second one [15] uses a recursive technique. Both approaches can lead to the same results but, although the latter "can be readily understood and applied by the typical polymer chemist" as stated by its promoters [16], the former approach has been preferred in this paper because its systematic step-by-step unfolding allows easy extensions to systems with components of various functionalities. More specifically, we refer to the work of Dušek and his coworkers ([1-4], for instance).

Our derivation given below is slightly more direct than what can be found in the literature for other systems, and connections with some previous results are, therefore, given. With our notations, the following polynomials of the dummy variable $z$

$$
\begin{aligned}
& F_{0 D}(z)=n_{D}(1-r x+r x z)^{2} \quad \text { and } \\
& F_{0 I}(z)=n_{I} \varphi(1-x+x z)^{3}+n_{I}(1-\varphi)(1-x+x z)^{4}
\end{aligned}
$$

are introduced first. They are such that the factors of $z^{k}$ in $F_{0 D}(z)$ and $F_{0 I}(z)$ give the molar fractions of 
diols and isocyanates with $k$ functional groups reacted, respectively. Taking the derivatives with respect to $z$ and normalizing by the value for $z=1$ gives

$$
\begin{aligned}
& F_{D}(z)=\frac{F_{0 D}^{\prime}(z)}{F_{0 D}^{\prime}(1)}=1-r x+r x z \quad \text { and } \\
& F_{I}(z)=\frac{F_{0 I}^{\prime}(z)}{F_{0 I}^{\prime}(1)}=\frac{3 \varphi(1-x+x z)^{2}+4(1-\varphi)(1-x+x z)^{3}}{4-\varphi},
\end{aligned}
$$

which lead to the two equations

$e_{D}=F_{D}\left(e_{I}\right) \quad$ and $\quad e_{I}=F_{I}\left(e_{D}\right)$

from which the functions $e_{D}(x)$ and $e_{I}(x)$ can be deduced. They define the probabilities that a reacted group of a diol or an isocyanate molecule has a finite bond-to-bond continuation. Consequently, $1-e_{D}(x)$ and $1-e_{I}(x)$ give the probabilities that a reacted group is connected to the gel. The solution to Eq. 12 is quite simple to obtain: Eq. 12 can be rewritten as $e_{D}=$ $F_{D}\left(F_{I}\left(e_{D}\right)\right)$, which leads to a second-degree equation with respect to $e_{D}$ when $e_{D}(x)$ (and, consequently, $\left.e_{I}(x)\right)$ being constant is excluded:

$$
\begin{aligned}
& 4(1-\varphi) r x^{4} e_{D}^{2}+[3(4-3 \varphi)-8(1-\varphi) x] r x^{3} e_{D} \\
& \quad+\left[4(1-\varphi) x^{2}+3(3 \varphi-4) x+6(2-\varphi)\right] r x^{2} \\
& \quad+\varphi-4=0 .
\end{aligned}
$$

Only one root is such that $e_{D}=0$ if $x=1$ when $r=1$ (i.e., all molecules belong to the gel for a perfect network):

$$
\begin{aligned}
& e_{D}(x)=1-\frac{3(4-3 \varphi) r x-\sqrt{\Delta}}{8(1-\varphi) r x^{2}} \quad \text { giving } \\
& e_{I}(x)=1-\frac{1-e_{D}(x)}{r x}
\end{aligned}
$$

with

$$
\Delta=(4-\varphi) r\left[16(1-\varphi)-3(4-5 \varphi) r x^{2}\right] .
$$

The gelation threshold corresponds to both $e_{D}(x)$ and $e_{I}(x)$ being equal to 1 :

$r_{g}=\frac{1}{6 x^{2}} \frac{4-\varphi}{2-\varphi}$,

which gives $r_{g}=0.43$ for full conversion $(x=1)$ when $\varphi=0.74$, with higher $r_{g}$ values if $x<1$. It can be checked that Eq. 16 corresponds to equation 3.57 of [17] if $\varphi=0$ : when no triisocyanate is present, the reac- tion between bifunctional diols and quadriisocyanates is formally similar to what happens in an epoxy resin. Moreover, when $\varphi=1$, Eq. 16 corresponds to equation 64 of [3], where $n_{A 1}$ and $n_{B 1}$ are taken equal to 0 (no monofunctional molecules).

Finally, replacing $x$ with $x\left(1-e_{I}\right)$ in $F_{0 D}(z)$ and with $x\left(1-e_{D}\right)$ in $F_{0 I}(z)$ gives

$$
\begin{aligned}
\widetilde{F}_{0 D}(z)= & n_{D}\left[1-r x+r x e_{I}+r x\left(1-e_{I}\right) z\right]^{2} \quad \text { and } \\
\widetilde{F}_{0 I}(z)= & n_{I} \varphi\left[1-x+x e_{D}+x\left(1-e_{D}\right) z\right]^{3} \\
& +n_{I}(1-\varphi)\left[1-x+x e_{D}+x\left(1-e_{D}\right) z\right]^{4},
\end{aligned}
$$

where the factor of $z^{k}$ is the molar fraction of molecules (diols in $\widetilde{F}_{0 D}(z)$, isocyanates in $\widetilde{F}_{0 I}(z)$ ) having $k$ reacted groups connected to chains with infinite continuation. It can be verified that when $\varphi=1$, Eq. 17 gives equations 66 and 67 of [3] with different notations.

Since it is defined by the molecules that have no bond with infinite continuation, the sol fraction is given by $\widetilde{F}_{0 D}(0)$ and $\widetilde{F}_{0 I}(0)$ for diols and isocyanates, respectively. Using Eq. 17, this leads to the following weight fraction of sol:

$$
\begin{aligned}
m_{s}= & \frac{1}{\bar{M}}\left[n_{D}\left(1-r x+r x e_{I}\right)^{2} M_{D}\right. \\
& +n_{I} \varphi\left(1-x+x e_{D}\right)^{3} M_{I 3}+n_{I}(1-\varphi) \\
& \left.\times\left(1-x+x e_{D}\right)^{4} M_{I 4}\right]
\end{aligned}
$$

which, with Eqs. 8 and 14, can be recast as

$$
\begin{aligned}
m_{s}= & m_{D} e_{D}^{2}+\left(1-m_{D}\right)\left(1-x+x e_{D}\right)^{3} \\
& \times\left[1-\frac{M_{I 4}}{\bar{M}_{I}}(1-\varphi) x\left(1-e_{D}\right)\right]
\end{aligned}
$$

in agreement with equation 68 of [3] when $\varphi=1$. This result is compared in Fig. 2 with the weight fraction of sol measured with PTMA-based polyurethanes. It can be observed that, although the experimental points have some discrepancy, the agreement is quite good when $x$ is between 0.96 and 1 . This suggests that the theory for network formation may be pertinent for our materials, and that almost full conversion was obtained. As a further illustration of the theory, it may be noted that the points where the curves meet the upper axis (weight fraction of sol equal to 1 ) correspond to $r_{g}$ given by Eq. 16, with a clear shift toward higher values when conversion is lower. 


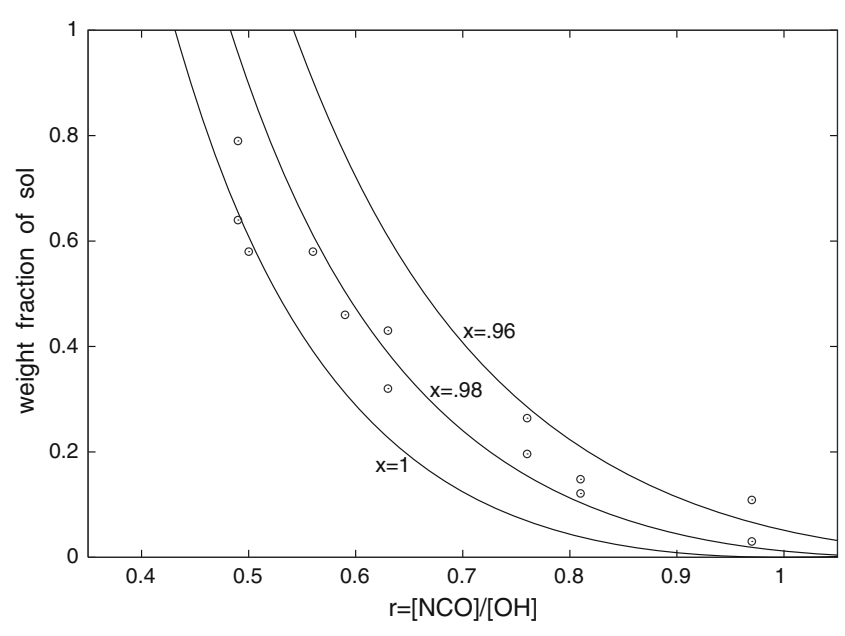

Fig. 2 Experimental results (open symbols) compared to the model predictions for the weight fraction of sol of the polyesterbased polyurethanes, using three conversion ratios

Prediction of the elastic shear modulus with previous models

Various theories have been developed to account for the elasticity of networks, and some will be considered here, which are of frequent use. These theories are often focused on nonlinear effects at finite strain, whereas our experimental results relate to the small strain shear modulus, and this allows using simplified versions of the complex expressions involved. Some of the quantities that these models require can be deduced from the theory of network formation described above, such as the molar fractions of 3-functional and 4-functional active nodes

$n_{3}=n_{I}\left[\varphi+4(1-\varphi)\left(1-x+x e_{D}\right)\right] x^{3}\left(1-e_{D}\right)^{3}$ and

$n_{4}=n_{I}(1-\varphi) x^{4}\left(1-e_{D}\right)^{4}$.

obtained by merely considering the factors of $z^{3}$ and $z^{4}$ in $\widetilde{F}_{0 I}(z)$. This provides the average functionality of active nodes $f=\left(3 n_{3}+4 n_{4}\right) /\left(n_{3}+n_{4}\right)$ and the number of elastically active chains per mole of initial mixture $\left(3 n_{3}+4 n_{4}\right) / 2$. Additional useful quantities can be deduced, like the total length of the elastically active chains, but most theories also require the fitting or evaluation of some parameters.

Figure 3 shows a typical example, where the phantom network model [7] has been applied, together with an extra term related to entanglements [8], like in [2]. Fitting has been used for the $r=1$ (and $x=1$ ) case only. The example is typical inasmuch as an upward curvature is obtained, which disagrees with our experimental results. Moreover, the curves are very representative of what is obtained with the elementary affine

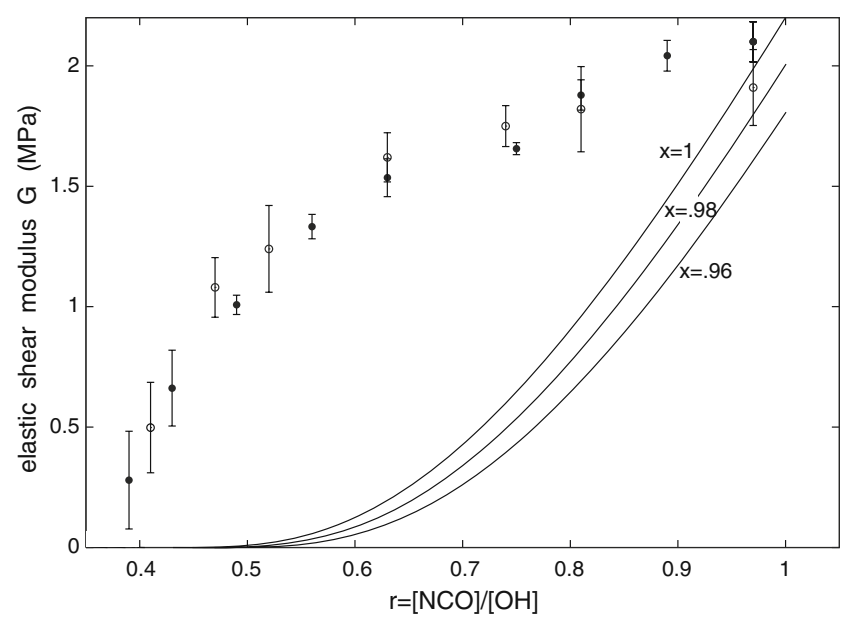

Fig. 3 Elastic shear modulus predicted by the theory of network formation combined with the phantom network model, with entanglements taken into account. Comparison with the experimental results obtained with the polyether-based $(1,000 \mathrm{~g} / \mathrm{mol}$, full symbols $)$ and the polyester-based $(1,035 \mathrm{~g} / \mathrm{mol}$, open symbols $)$ polyurethanes

theory of rubber elasticity [6] (no fitting required), and with such elaborate models as the diffused-constraint model of Kloczkowski et al. [9] and the constrainedchain model of Erman and Monnerie [10]. The latter two theories predict a shear modulus that is up to 1.5 times the modulus given by the affine model for a tetrafunctional network, which unfortunately is not enough to cover the gap with experimental results in Fig. 3 for imperfect networks. Curves very similar to those in Fig. 3 were also obtained with the slip-link theory of Edwards and Vilgis [11], with various combinations of the required parameters $\eta, \alpha$ and average number of slip links per chain.

\section{Discussion}

The experimental results that were obtained in this study have been shown to be in reasonably good agreement with the statistical theory of network formation as far as the sol fraction is considered, but the measured elastic shear modulus could not be reproduced by combining this theory with rubber elasticity models. This combination predicts an upward curvature of the $G(r)$ plot, which is found in accordance with experimental results obtained on different systems by Ilavsý et al. [4], for instance, with triisocyanate and diols. In this comparison, it should be kept in mind that $r$ is defined as $[\mathrm{OH}] /[\mathrm{NCO}]$ in the latter paper, and that log scales are used in $G$ plots. Nevertheless, the linear decreasing trend obtained in Figure 3 of [4], for instance, can be interpreted immediately as an upwardly curved $G(r)$ plot with our notations. In contrast, our experimental 
results are consistent with observations made by Burel et al. [18] on a similar system. Using a diol (hydrogenated hydroxytelechelic polyisoprene) and an isocyanate with an average functionality of $\bar{f}_{w}=3.43$, they did not measure the elastic shear modulus but rather deduced the crosslink density $v_{e}$ by applying the Flory-Rehner [19] theory to swelling measurements on the polyurethane networks obtained. Interpreting the results with the affine theory for rubber elasticity suggests a $G(r)$ plot with a downward curvature, like the $v_{e}$ vs $[\mathrm{NCO}] /[\mathrm{OH}]$ plot shown in Figure 8 of [18].

In order to interpret our experimental results, more elaborate and recent models for network formation could have been considered. Accounting for intramolecular reactions as proposed by [20], for instance, could be a valuable refinement, but it would have lead to a gel point value higher than that given by Eq. 16, which was found to be slightly higher than the observed one ( 0.43 vs less than 0.4, see Fig. 1). It has rather been preferred here to keep with the standard model for network formation and explore non-standard interpretations of its results in terms of elastically active chains to be used in the elementary theory of rubber elasticity.

Let us assume, for instance, that all the isocyanates in the gel with three or four reacted groups are active nodes. A possible interpretation of this assumption could be that all pendant chains (attached to the gel through a single bond with finite continuation) are active actually because they are entangled in each other, for instance. The molar fraction of isocyanates that have tri- or quadri-reacted is directly obtained from Eq. 10, and the fractions that belong to the sol can be deduced from Eq. 17, as explained above. The differences give the numbers of nodes with three and four reacted groups in the gel obtained from one mole of mixture:

$n_{3}^{\prime}=n_{I}[4-3 \varphi-4(1-\varphi) x]\left(1-e_{D}^{3}\right) x^{3} \quad$ and

$n_{4}^{\prime}=n_{I}(1-\varphi)\left(1-e_{D}^{4}\right) x^{4}$.

Assuming that all these nodes are active, these relations provide the number of active chains $\left(3 n_{3}^{\prime}+4 n_{4}^{\prime}\right) / 2$, and the affine model for rubber elasticity leads to the elastic shear modulus

$G=v_{e} R T=\frac{3 n_{3}^{\prime}+4 n_{4}^{\prime}}{2} \frac{\rho}{\bar{M}} R T$.

Such a theory increases the density of active chains in the network and, therefore, the elastic shear modulus, and Fig. 4 shows that the effect is such that the curvature of the $G(r)$ plot is changed to downward. This is in better agreement with the experimental results, but the predictions are still too low for small $r$ values.

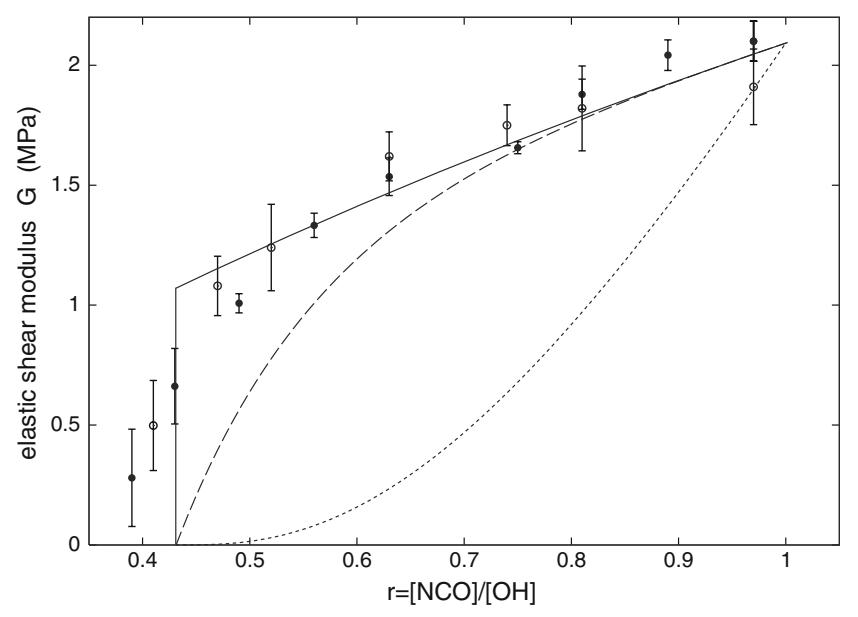

Fig. 4 Predictions, for full conversion $(x=1)$, of the standard model (dotted line), of the model assuming that all three- and four-reacted isocyanates in the gel are active (broken line), and of the model assuming that all three- and four-reacted isocyanates in the system are active (unbroken line). Comparison with the experimental results for the 1,000 - and $1,035-\mathrm{g} / \mathrm{mol}$ diols

An ultimate increase of the elastic shear modulus can be obtained by a very simple assumption, considering that all three- or four-reacted isocyanates in the system are elastically active nodes. This assumes implicitly that the molecules in the sol that contain at least one isocyanate with three or four groups reacted are physically connected to the gel and contribute to the elastic properties measured, whereas the solvent is able to disconnect them when the sol fraction is measured. In these conditions, the model for network formation is used only for predicting the gel point, after which the degree of conversion $x$ immediately leads to the molar fractions of three- and four-reacted isocyanates, using Eq. 10:

$n_{3}^{\prime \prime}=n_{I}(4-3 \varphi) x^{3} \quad$ and $\quad n_{4}^{\prime \prime}=n_{I}(1-\varphi) x^{4}$.

Combined with Eq. 22 to obtain the elastic shear modulus, this leads to the upper curve shown in Fig. 4, which is in good agreement with the experimental results. The jump for $r=r_{g}$ is due to the three- and four-reacted isocyanates that are already present in the sol for $r<r_{g}$ and which suddenly belong to the gel when the latter appears. When this simple theory is applied to the 2,000- and 4,000-g/mol diols, Fig. 5 shows that it still predicts rather well the observed values of the shear modulus.

These results indicate that the critical underestimation of the number of elastically active chains for the systems considered here can be amended by a different counting. This allows preserving the simplicity of the standard theories for both network formation and 


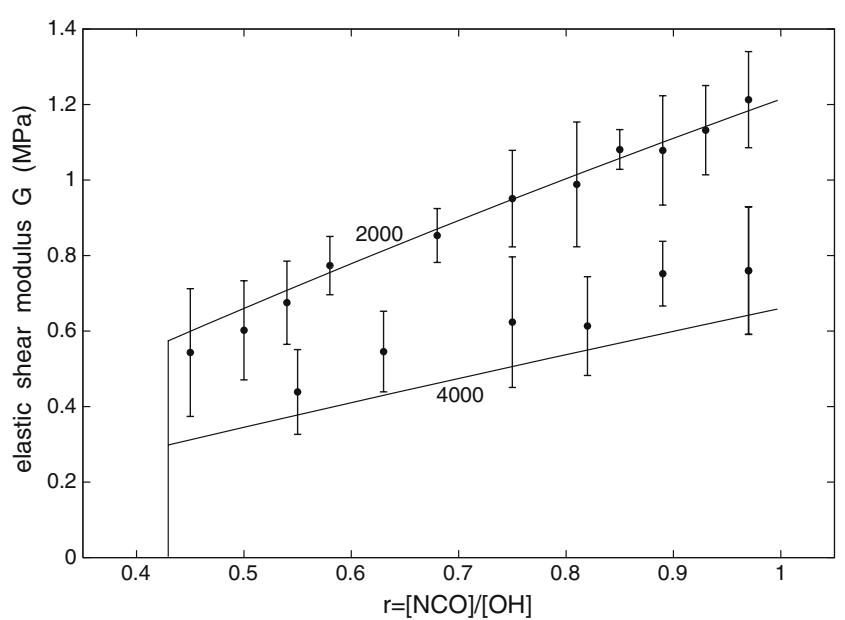

Fig. 5 Predictions of the elastic shear modulus given by the simple theory (at full conversion, $x=1$ ) for 2,000- and 4,000$\mathrm{g} / \mathrm{mol}$ diols, compared with the experimental results

rubber elasticity, with a good concordance between model and experiments.

\section{Conclusions}

Polyurethane networks have been prepared from a mix of tri- and quadriisocyanate and from two types of diols, either polyether-based (with molar masses of $1,000,2,000$, and $4,000 \mathrm{~g} / \mathrm{mol}$ ) or polyester-based $(1,035 \mathrm{~g} / \mathrm{mol})$. The ratio $r=[\mathrm{NCO}] /[\mathrm{OH}]$ has been varied but kept below 1 . The weight fraction of sol has been measured, as well as the elastic shear modulus $G$ of the gels. It has been found that the statistical theory of network formation predicts a weight fraction of sol in agreement with the experimental results, but its combination with various theories of rubber elasticity disagrees significantly with the elastic modulus measured: the $G(r)$ plot has the wrong curvature.

In an attempt to keep both the theory of network formation and the elementary theory of rubber elasticity, the definition of elastically active nodes has been modified. Taking into account all the three- and fourreacted isocyanates in the gel leads to an inversion of the curvature of the $G(r)$ plot, which brings predictions significantly closer to experimental results. An even better agreement is obtained when all three- and fourreacted isocyanates in the system are considered as active nodes, which is an upper bound of the actual number of active nodes in a given system. Of course, further work is needed to determine what is specific in the systems considered that induces the downward curvature observed in the $G(r)$ plot, which has also been obtained by other authors.
Acknowledgements This work was supported by grant JC0543403 from the French Agence Nationale de la Recherche. The authors are grateful to $\mathrm{C}$. Clin for his contribution to the experimental work.

\section{References}

1. Dušek K (1989) Formation and structure of networks from telechelic polymers: theory and application to polyurethanes. In: Goethals E (ed) Telechelic polymers: synthesis and applications. CRC, Boca Raton, pp 289-360

2. Ilavský M, Dušek K (1983) The structure and elasticity of polyurethane networks: 1 . Model networks of poly(oxypropylene) triols and diisocyanate. Polymer 24:981990

3. Dušek K, Dušková M, Fedderly JJ, Lee GF, Hartmann B (2002) Polyurethane networks with controlled architecture of dangling chains. Macromol Chem Phys 203:1936-1948

4. Ilavský M, Šomvársky J, Bouchal K, Dušek K (1993) Structure, equilibrium and viscoelastic mechanical behaviour of polyurethane networks based on triisocyanate and poly(oxypropylene) diols. Polym Gels Netw 1:159-184

5. Gordon M (1962) Good's theory of cascade processes applied to the statistics of polymer distributions. Proc R Soc Lond A268:240-259

6. Kuhn W (1923) Beziehungen zwischen Molekülgröße, statistischer Molekülgestalt und elastischen Eigenschaften hochpolymerer Stoffe. Kolloid-Z 76:258-271

7. Flory PJ (1976) Statistical thermodynamics of random networks. Proc R Soc Lond A351:351-380

8. Langley NR (1968) Elastically effective strand density in polymer networks. Macromolecules 1:348-352

9. Kloczkowski A, Maark JE, Erman B (1995) A diffusedconstraint theory for the elasticity of amorphous polymer networks. 1. Fundamentals and stress-strain isotherms in elongation. Macromolecules 28:5089-5096

10. Erman B, Monnerie L (1989) Theory of elasticity of amorphous networks: effect of constraints along chains. Macromolecules 22:3342-3348

11. Edwards SF, Vilgis T (1986) The effect of entanglements in rubber elasticity. Polymer 27:483-492

12. Pegoraro M, Penati A, Zanderighi L (1986) Polyurethane membrane for gas fractionation. J Membr Sci 27:203-214

13. Flory PJ (1941) Molecular size distribution in three dimensional polymers. I. Gelation. II. Trifunctional branching units. III. Tetrafunctional branching units. J Am Chem Soc 63:3083-3100

14. Stockmayer WH (1943) Theory of molecular size distribution and gel formation in branched-chain polymers. J Chem Phys 11:45-55

15. Miller DR, Macosko CW (1976) A new derivation of postgel properties of network polymers. Macromolecules 9:206-211

16. Macosko CW, Miller DR (1976) A new derivation of average molecular weights of nonlinear polymers. Macromolecules 9:199-206

17. Pascault JP, Sautereau H, Verdu J, Williams RJJ (2002) Thermosetting Polymers. Marcel Dekker, New York

18. Burel F, Feldman A, Bunel C (2005) Hydrogenated hydroxyterminated polyisoprene (HHTPI) based urethane network: network properties. Polymer 46:483-489

19. Flory PJ, Rehner JJ (1943) Statistical mechanics of crosslinked polymer networks. II Swelling. J Chem Phys 11:521526

20. Cail JI, Stepto RFT (2007) The gel point and network formation-theory and experiment. Polym Bull 58:15-25 